\section{WEB WATCH}

Genetic testing 1, 2, 3

The identification of a disease gene usually provides one immediate and practical benefit to individuals and families affected by the disease a diagnostic test that can be used, in conjunction with genetic counselling, for carrier detection, prenatal diagnosis and so on. But how is this information publicized so that affected families, and their healthcare providers, know what help is available? In the United States, two publicly funded web sites that aim to meet this need are GeneClinics and GeneTests.

The information in

GeneClinics is organized into disease profiles. For each disease, information is structured in the same way - diagnosis, management, genetic counselling and molecular genetics are a selection of the topics that are covered. Links are also provided to patient support groups. The disease profiles are written by experts, peerreviewed, referenced (with links to PubMed and OMIM) and are regularly updated.

The companion site,

GeneTests, brings together information on where to find the required genetic services. Two types of service are covered laboratories offering genetic testing and genetic clinics providing diagnosis, management and counselling. A slideshow is also provided for use by genetics professionals for educational purposes.

Participation by laboratories or clinics in GeneTest is voluntary, so the site is not comprehensive, but with around 500 laboratories and 900 clinics listed the site is a valuable first port of call.

Together, GeneClinics and GeneTests are part of the public face of genetics. Even if you don't need their services, the sites provide a clear indication of how much genetics has achieved and how much it has to offer.

Mark Patterson

PLANT GENETICS

\title{
If winter comes, will flowers follow?
}

In Nature's shop window, flowers aren't on display all year round, as a plant's flowering time depends on the precise interaction between genetic and environmental conditions. After decades of studying the flowering properties of plants at the physiological level, a molecular approach has become possible, as exemplified by the work of Johanson and colleagues. Their recent study shows that the natural variation in flowering time in Arabidopsis thaliana is determined, in large part, by polymorphisms at a single locus.

Some plants can flower only after a few weeks of cold treatment, presumably as an insurance against blossoming in an ungrateful winter climate. In Arabidopsis, this requirement for cold, called vernalization, segregates as a single-gene trait that maps to the FRIGIDA (FRI) locus. Not all plants have a vernalization requirement - this late-flowering character has been bred, to our advantage, into many crop plants to produce winter-spring varieties. Early-flowering plants, in contrast, do not need a cold spell to burst into flower.

Johanson et al. cloned the FRIGIDA gene, and found that it encodes a protein of unknown function. They then examined the allelic variation at this locus in Arabidopsis ecotypes (sub-populations that are adapted to specific environmental conditions), in the hope of finding polymorphisms that determine the early/late flowering phenotype. Using these polymorphisms (deletions or amino-acid substitutions), they showed that the early-flowering plants have evolved independently several times from the late-flowering plants, which contain the ancestral form of FRI. 'Early' alleles behave similarly to presumed null alleles, suggesting that natural early alleles have lost FRI function.
Curiously, although one group of plants was early flowering, it seemed to have a functional FRI gene. Some of the early-flowering plants in this ecotype had a recessive mutation in a second gene, FLOWERING LOCUS C (FLC), whose RNA is positively controlled by FRI. One other plant of this group had no obvious mutation in either FRI or FLC, indicating the involvement of other genes.

The fact that the early-flowering trait has emerged more than once led the authors to investigate the selective pressures that drive the variation in flowering times. No significant correlation was found in this study between flowering time and environmental conditions, such as mean temperature - the main influence on the distribution of the ecotypes seems to be man's intervention. Human disturbance continuously exposes plants to new environments, to which plants are forced to adapt.

The genes responsible for transmitting the cold signal to the plant have yet to be found, but their discovery will certainly come from studying different Arabidopsis collected from the wild. This is because the ecotypes used in the lab so far are the most rapid-cycling ones (geneticists are an impatient bunch), which no longer need the cold to flower.

Tanita Casci

\section{(0) References and links}

ORIGINAL RESEARCH PAPER Johanson, $U$. et al. Molecular analysis of FRIGIDA, a major determinant of natural variation in Arabidopsis flowering time. Science 290, 344-347 (2000)

FURTHER READING Soppe, W. J. J. et al. The late flowering phenotype of fwa mutants is caused by gain-of-function epigenetic alleles of a homeodomain gene. Mol. Cell 6, 791-802 (2000) WEB SITE Caroline Dean's lab

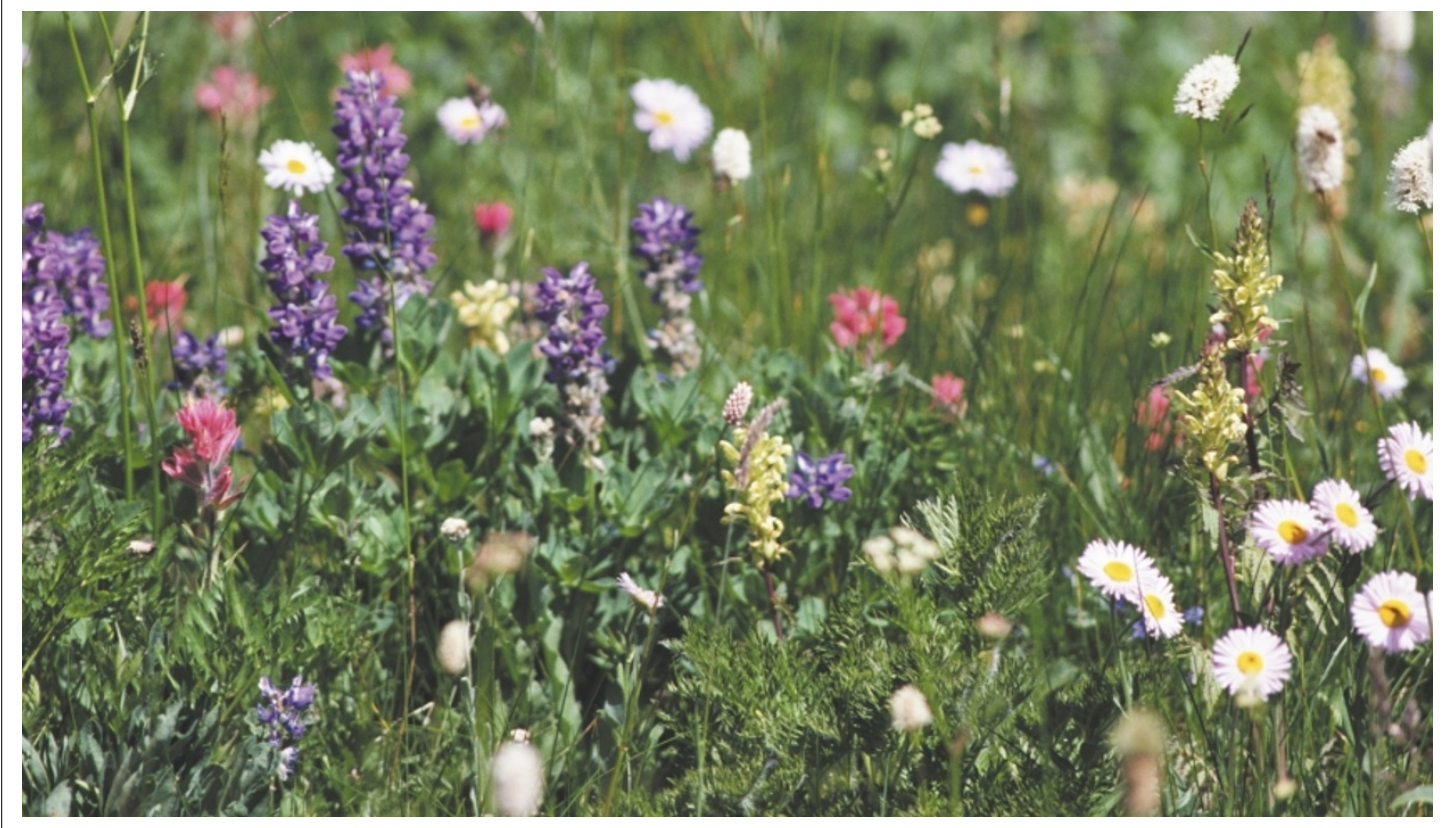

